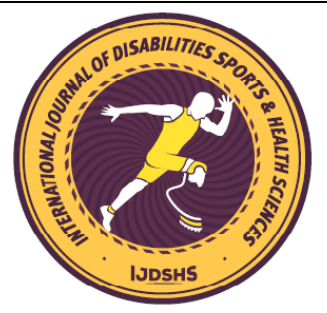

\title{
Segmental Musculoskeletal Examinations of Injured Adolescent Ballet Dancers Mimic a Decreased Strength, Anaerobic Power and Endurance Performance
}

Gökhan UMUTLU*1' ${ }^{\text {(D) }}$ and Yağmur ARINLI ${ }^{2}$ iD

\author{
${ }^{* 1}$ Mersin University, Faculty of Sports Science, Turkey \\ ${ }^{2}$ Mersin University, State Conservatory, Turkey \\ *Corresponding author: gokhannumutluu@gmail.com
}

\begin{abstract}
The primary purpose of this research was to gather information on whether overload placed upon lower extremity muscles during aerobic dance-only classes induce negative adaptations on muscular strength, endurance and, anaerobic capacity due to the inadequate strength and conditioning programs. The second purpose was to determine whether pre-season screenings of injured dancers showed drastic changes in isokinetic mean work, power, and moments before injury occurrence to identify predisposing factors compared to healthy counterparts. Ten healthy and 11 injured adolescent female ballet dancers who experienced anterior cruciate ligament injury during ballet performance participated in this study. Anthropometric measurements, anaerobic power, muscle strength and endurance performance scores were recorded using preliminary testing sessions. Significant correlations were occurred between fatigue parameters of extensor muscles and the decline in knee extension mean work, power, and moment $(\mathrm{r}=.831, \mathrm{p}<.001 ; \mathrm{r}=.961, \mathrm{p}<.001 ; \mathrm{r}=.969, \mathrm{p}<.001)$. Fatigue index of flexor muscles was also positively correlated with the decline in mean work, power, and moment parameters of knee flexor muscles at $1-5$ to last 5 reps $(\mathrm{r}=.818, \mathrm{p}<.001 ; \mathrm{r}=.837, \mathrm{p}<.001 ; \mathrm{r}=.931, \mathrm{p}<.001)$, respectively. As a result, segmental musculoskeletal examinations on anaerobic power, muscle strength and endurance performance revealed that dancers performing aerobic dance only classes are prone to suffer lower extremity injuries and muscular characteristics of ankle, knee and hip muscles also predispose a decreased strength, anaerobic power and endurance performance following an anterior cruciate ligament injury.
\end{abstract}

Keywords

Ballet, Strength, Anaerobic Power, Endurance, Range of Motion

\section{INTRODUCTION}

Ballet is a performing art form that requires enhanced muscle strength, aerobic and anaerobic capacity performing physically demanding and purposefully selected sequences of human movements. Especially in the classical ballet, which is formed with the execution of a sequence of steps, dancers are not only expected to show their performance skills coordinated with music but also must the techniques be shown with grace, poise, and understanding the nature of ballet (Guidetti et al., 2007). Due to the demand for specific movements during ballet performance, dancers demonstrate a greater range of motion and strength at the hip joint compared to the counterparts from other sports disciplines while they have weaker upper body, torso, hamstrings, and quadriceps strength (Teitz, 2000). Despite this fact, elected parts of the body are subjected to loads greater than those to which they are accustomed to performing purposefully selected sequences of movements. In this regard, an overload, a possible strength discrepancy, or a bilateral strength asymmetry in the lower extremity muscles may lead to serious injuries due to the improper alignment of the body during some specific ballet movements. 
One of the most common misconceptions in ballet curriculum is that aerobic dance classes are thought to be sufficient to meet all physical and physiological demands of dancers even during the recovery period following an injury despite the fact that orthopedic rehabilitation can no longer do without strength and endurance training either (Stracciolini et al., 2016). However, the traditional ballet curriculum does not contain additional strength training programs in order to avoid undesirable hypertrophy due to the aesthetic demands of ballet (Twitchett et al., 2009). This common misconception is not surprising considering the assumptions about dance conditioning which are thought to be "myths" and have no basis, in fact.

Monitoring the muscular strength status of dancers can aid to minimize the risk of injury and enables the teachers to detect any progression towards negative health outcomes associated with poor performance since the insufficient amount of strength exercise in ballet curriculum may also increase the potential risk of injuries resulted from strength deficiency (Koutedakis et al., 2007). Most of the specific ballet movements, as well as turnout, more readily achieved in the young dancers depends on the soft tissue allowance at the hip joint and this excessive load may also cause serious injuries and significant damage to these joints due to inadequate strength level at this ages. Additionally, most teachers encourage the students to turn out only from the hip angles due to the form of the movement. However, a study suggests that on average, $60 \%$ of turnout was created by the outward rotation of the hip, and 30\% from the ankle, and the remaining percentage created by the tibia and knee joint (Grossman et al., 2005). The multiple structures of these specific movements in ballet require extra precision to understand the demands of complex movements which are necessary to optimize turnout in young ballet dancers. The hip and ankle joints have an essential aspect in the control of balance. The main function of the ankle joint is the provision of the balance control against postural disturbance, absorption of shock during gait, and movement of the lower extremity (Mertz and Docherty, 2012). Therefore, a normal range of motion in ankle joint and muscular strength are important components to maintain the postural sway during the movements (Yu-Hyung et al., 2013; Chi-Hung et al., 1994).
Due to the increased demand for enhanced muscle strength, endurance, and anaerobic power capacity especially in the lower extremity muscles in ballet, the purpose of this study was two-fold. The first scope of this study was to evaluate isokinetic hip, knee, ankle plantar, and dorsiflexion peak moment strengths at different angular velocities to observe the effect of overload placed upon the lower extremity muscles during aerobic dance only on strength development. Also, the second rationale of this study was to evaluate whether the post operation screening of injured dancers showed drastic changes in mean work, power, and moments compared to pre-injury screenings.

\section{METHODS}

\section{Participants:}

Before participating in the study, all subjects gave a written informed consent according to Mersin University Local Ethical Committee (Chairperson: Dr. Bahar TUNÇTAN, protocol number: 632842, date of approval: 11.01.2018) and all were familiarized with the testing procedures and informed about equipment before all test sessions. Ten non-injured control and 11 injured female adolescent ballet dancers who experienced anterior cruciate ligament injury during ballet classes volunteered to participate in this study. Based on the results of the knee MRI examination, the participants were diagnosed with grade II degeneration in the dorsal horn of the medial meniscus. The dorsal horn of the lateral meniscus was found partially extended into the intercondylar notch compatible with the bucket handle tear in the MRI examinations. An abnormal contour and heterogeneous edema signal increase was noted along anterior cruciate ligament and medial collateral ligament consistent with grade II sprain. The level of knee joint fluid was slightly increased and an effusion compatible with suprapatellar plica was also noted in suprapatellar fossa in all screenings.

\section{Test Procedures:}

All participants gave written informed consent prior to participating with the study approved by Institutional Review Board in accordance with the ethical standards of the Helsinki Declaration. All participants were informed about equipment and familiarized to the 
experimental procedures before they underwent testing sessions. The anthropometric parameters of participants were expressed as Mean \pm SD. The anthropometric parameters (body fat mass, lean body mass, body weight) were assessed using Bioelectrical impedance analysis (Tanita 418-MA Japan) prior to Cybex isokinetic test sessions. Heights of the participants were measured with a stadiometer in the standing position (Holtain Ltd. U.K.). All test sessions were conducted with 48 hours intervals.

\section{$60 \%$ and $180 \%$ Isokinetic Peak Moment Knee Muscle Strength Testing:}

Prior to the isokinetic knee extension and flexion peak moment measurement, the participants were seated in the upright position with the hips flexed at an angle of $90^{\circ}$ and through pelvic and thigh straps the hips and thighs of participants were stabilized. Before the test session participants performed dynamometer trials over a series of 10 submaximal repetitions (about $50 \%$ maximum voluntary contraction), both during flexion and extension at $180 \%$ s.

Following on the warm-up session, they performed 5 maximal bilateral knee extension repetitions in an isokinetic test protocol at an angular velocity of $60 \% \mathrm{~s}$ and $180 \%$ s to determine isokinetic peak moment strength. The participants were asked to perform as quickly as possible and at a maximal effort. They were also told to grasp the handles at the sides of the chair throughout the warm-up and the test. Gravity correction was implemented prior to the isokinetic test protocol session. The participants underwent the same protocol for both legs during all isokinetic testing sessions. The hamstrings-to-quadriceps strength ratios (H/Q ratio/eccentric to concentric), calculated as the hamstrings peak moment divided by the peak moment of the quadriceps within the same limb.

\section{Isokinetic Muscular Endurance Capacity Testing Protocol:}

Upon arrival to the laboratory for the next visit, the participants underwent same warm-up routine and performed 50 maximal bilateral knee extension repetitions at an angular velocity of $180^{\circ}$ s to determine muscle endurance capacity. They were asked to perform as quickly as possible but typically not at a maximal effort. The mean work, power and moment of knee extension and flexion moment for repetitions 1-5, 21-25 and the last five repetitions of endurance test was determined to use in calculations. For example, the decline in endurance performance during the 50 repetitions of reciprocal knee contractions was determined: (MME of first 5 reps - MME of last 5 reps / MME of first 5 reps). This formula was also used to determine the mean moment, work, and average power for repetitions $21-25$ or the final five repetitions relative to the first five repetitions. The participants underwent the same protocol for both legs during all isokinetic testing sessions. The fatigue rate was calculated as follows (Kawabata et al., 2000).

(Maximum moment of the first 5 knee bends the maximum moment of the last 5 knee bends) / maximum moment of the first 5 knee bends $\mathrm{x} 100$

\section{$60 \%$ and $180 \%$ Isokinetic Peak Moment Ankle Muscle Strength Testing:}

In the following test session, ballet students underwent isokinetic measurement at an angular velocity of $60 \% \mathrm{~s}$ and $180 \% \mathrm{~s}$ in the supine position to determine absolute $(\mathrm{Nm})$ and relative $(\mathrm{Nm} / \mathrm{kg})$ ankle plantar flexion and dorsiflexion peak moment. Additional straps were used to stabilize the participant during ankle testing. Ankle ROM was determined as the participant's maximum plantar and dorsiflexion. After the standard joint specific warm-up and 2 minutes of rest, ballet students performed 5 maximal concentric plantar flexion repetitions at $60 \% \mathrm{~s}$ and $180 \%$ s. At the end of each repetition, participants were told to relax and the ankle was returned to the starting position. Participants performed 5 concentric maximal dorsiflexion repetitions at $60 \%$ s and $180 \%$ s.

\section{$60 \%$ and $180 \%$ Isokinetic Peak Torque Hip Muscle Strength Testing:}

On the fourth visit to the laboratory, participants laid supine on the dynamometer chair with the chair back completely flattened to measure hip flexion/extension peak moment strength at an angular velocity of $60 \%$ s and $180 \%$ s. The tested hip was at $0^{\circ}$ of flexion, with $90^{\circ}$ of knee flexion, and secured into a brace. The tested thigh was strapped to the dynamometer pad at femur level. The non-tested thigh was stabilized to the dynamometer chair at $0^{\circ}$ of hip flexion. The pelvis and trunk were strapped to the dynamometer chair to prevent undesirable movements 
throughout the test. Gravitational corrections were made prior to all test sessions to avoid the effect of

\section{Wingate (WAnT) Anaerobic Power Performance Assessment:}

electronically braked Monark 864. All participants performed a standardized warm-up, which consisted of 4 min of steady state pedaling at 60 revolutions per minute (rpm), interspersed with three 2-to 3-s periods of all-out cycle sprints preceded each WAnT. Seat height was adjusted to each participant's satisfaction, and clips with straps were used to prevent the feet from slipping off the pedals. Each participant cycled $30 \mathrm{~s}$ against constant resistance. For female participants resistance was set to $0.53 \mathrm{~N} \cdot \mathrm{m}$ per kilogram body weight. Participants were instructed to pedal as fast as possible throughout the test period and were verbally encouraged throughout the test. In each test maximal power output, mean power output, minimal power output, and fatigue index were measured. All power output measurements are based on $5 \mathrm{~s}$ averages that were calculated by the WAnT computer software and were reported in watts per kilogram $(\mathrm{W} / \mathrm{kg})$. Maximal power output (peak power) was calculated from the highest $5 \mathrm{~s}$ work output. Mean power output, which reflects the anaerobic capacity, was calculated as the mean power output throughout the $30 \mathrm{~s}$ of the test. Minimal power output was calculated as the lowest $5 \mathrm{~s}$ work output. Fatigue index was calculated as the percentage of power output drop from the maximal power output throughout the test.In each test maximal power output, mean power output, minimal power output, and fatigue index were measured. All power output measurements are based on $5 \mathrm{~s}$ averages that were calculated by the WAnT computer software and were reported in watts per kilogram $(\mathrm{W} / \mathrm{kg}$ ). Maximal power output (peak power) was calculated from the highest $5 \mathrm{~s}$ work output. Mean power output, which reflects limb weight on moment production.

On the fifth visit to the laboratory each participant underwent a Wingate test (WAnT) on an

the anaerobic capacity, was calculated as the mean power output throughout the $30 \mathrm{~s}$ of the test. Minimal power output was calculated as the lowest $5 \mathrm{~s}$ work output. Fatigue index was calculated as the percentage of power output drop from the maximal power output throughout the test.

\section{Statistical Analysis:}

Descriptive statistics were used to summarize data whilst Spearman's Rank-Order Correlation analysis determined the correlations of the variables at a 95\% confidence interval. A nonparametric Mann-Whitney U test was used to compare inter-group and intra-group differences. The level of statistical significance was set at $\mathrm{p}<.05$ and $\mathrm{p}<.001$ for all comparisons. The statistical analysis was performed with SPSS version 20.0 (SPSS Inc., Chicago, IL, USA).

\section{RESULTS}

Ten healthy (age:13.36 \pm 0.17 years; height: $160.10 \pm 3.27 \mathrm{~cm}$, body weight: $47.14 \pm 6.25 \mathrm{~kg}$, percent body fat: $20.11 \pm 5.55 \%$, lean body mass: $37.30 \pm 4.35 \mathrm{~kg}$, years of sports participation: $5.13 \pm 4.26$ years, years on pointe: $2.01 \pm 1.21$ years) and 11 injured (age:13.15 \pm 2.35 years; height: $162.33 \pm 5.24 \mathrm{~cm}$, body weight: $48.45 \pm 6.56 \mathrm{~kg}$, percent body fat: $24.31 \pm 6.65 \%$, lean body mass: $35.30 \pm 2.57 \mathrm{~kg}$, years of sports participation: $5.11 \pm 1.25$ years, years on pointe: $2.12 \pm 3.01$ ) female adolescent ballet dancers voluntarily participated in this study. Descriptive data of ankle range of motion parameters of participants are shown as mean \pm standard deviation $(M e a n \pm S D)$ in Table 1.

Table 1. Range of motion parameters of participants during plantar flexion and dorsiflexion measurements at $60 \%$ and $180 \%$ angular velocity (Mean \pm SD).

\begin{tabular}{|c|c|c|c|c|}
\hline Variable $(n=21)$ & $\begin{array}{c}60^{\circ} / \mathbf{s} \\
\operatorname{Right}^{\left({ }^{\circ}\right)}\end{array}$ & $\begin{array}{c}60^{\circ} / \mathrm{s} \\
\text { Left }^{\left({ }^{\circ}\right)}\end{array}$ & $\begin{array}{c}180^{\circ} / \mathrm{s} \\
\left.\operatorname{Right}^{\circ}{ }^{\circ}\right)\end{array}$ & $\begin{array}{c}180^{\circ} / \mathrm{s} \\
\text { Left }^{\circ}\left({ }^{\circ}\right)\end{array}$ \\
\hline Plantar Flexion & $51.45 \pm 11.50$ & $51.00 \pm 11.23$ & $50.36 \pm 12.62$ & $47.18 \pm 9.66$ \\
\hline Dorsiflexion & $23.36 \pm 7.28$ & $19.73 \pm 8.09$ & $19.55 \pm 12.08$ & $20.91 \pm 8.84$ \\
\hline
\end{tabular}


Comparison of isokinetic strength parameters of participants:

Based on the results of the Mann Whitney U analysis there was a significant difference in $60 \% \mathrm{~s}$ dorsiflexion peak moment parameters between dominant and non-dominant limbs $(\mathrm{U}=23.00, \mathrm{Z}=$ $2.474, \mathrm{p}<0.05)$. However, no significant difference occurred between $180 \%$ ankle plantar and dorsiflexion, hip and knee isokinetic peak moment parameters of the participants (Table 2).

Table 2. Isokinetic plantar flexion and dorsiflexion, knee and hip extension-flexion peak moment parameters $(\mathrm{n}=21)$.

\begin{tabular}{|c|c|c|c|c|c|c|c|c|}
\hline & $\begin{array}{c}\mathbf{6 0} / \mathbf{s} \\
\text { Right } \\
(\mathrm{Nm}) \\
\end{array}$ & $\begin{array}{l}\mathbf{6 0} / \mathbf{s} \\
\text { Left } \\
(\mathrm{Nm}) \\
\end{array}$ & $\begin{array}{c}\text { 180\%/s Righ } \\
(\mathrm{Nm})\end{array}$ & $\begin{array}{r}180^{\circ} / \mathrm{s} \\
\text { Left } \\
(\mathrm{Nm}) \\
\end{array}$ & $\begin{array}{c}\mathbf{6 0} \% \text { s } \\
\text { Right } \\
(\mathrm{Nm} / \mathrm{kg})\end{array}$ & $\begin{array}{c}\mathbf{6 0} / \mathbf{s} \\
\text { Left } \\
(\mathrm{Nm} / \mathrm{kg}) \\
\end{array}$ & $\begin{array}{c}\mathbf{1 8 0} / \mathrm{s} \\
\text { Right } \\
(\mathrm{Nm} / \mathrm{kg}) \\
\end{array}$ & $\begin{array}{c}180^{\circ} / \mathbf{s} \\
\text { Left } \\
(\mathrm{Nm} / \mathrm{kg}) \\
\end{array}$ \\
\hline$\overline{\mathbf{P F}}$ & $48.18 \pm 15.99$ & $43.91 \pm 17.09$ & $39.73 \pm 13.57$ & $41.36 \pm 10.19$ & $1.06 \pm 0.39$ & $0.95 \pm 0.30$ & $0.89 \pm 0.33$ & $0.93 \pm 0.29$ \\
\hline DF & $24.18 \pm 15.65^{*}$ & $34.45 \pm 13.82 *$ & $26.10 \pm 11.73$ & $28.91 \pm 10.34$ & $0.54 \pm 0.34$ & $0.80 \pm 0.46$ & $0.58 \pm 0.26$ & $0.64 \pm 0.23$ \\
\hline KE & $114.91 \pm 21.09$ & $115.82 \pm 24.26$ & $92.00 \pm 17.87$ & $94.10 \pm 20.02$ & $2.56 \pm 0.64$ & $2.57 \pm 0.71$ & $2.04 \pm 0.51$ & $2.08 \pm 0.53$ \\
\hline $\mathbf{K F}$ & $60.19 \pm 8.61$ & $58.82 \pm 10.01$ & $49.00 \pm 9.63$ & $48.73 \pm 11.44$ & $1.32 \pm 0.20$ & $1.30 \pm 0.28$ & $1.07 \pm 0.19$ & $1.07 \pm 0.26$ \\
\hline HE & $132.91 \pm 32.81$ & $125.18 \pm 31.47$ & $100.00 \pm 23.6$ & $89.73 \pm 22.94$ & $2.96 \pm 0.88$ & $2.80 \pm 0.91$ & $2.22 \pm 0.67$ & $2.01 \pm 0.74$ \\
\hline HF & $66.36 \pm 16.00$ & $58.00 \pm 14.11$ & $61.36 \pm 8.33$ & $63.10 \pm 11.66$ & $1.45 \pm 0.29$ & $1.28 \pm 0.31$ & $1.34 \pm 0.12$ & $1.42 \pm 0.41$ \\
\hline
\end{tabular}

Mean \pm SD. Note. PF: Plantar flexion, DF: Dorsiflexion, KE: Knee extension, KF: Knee flexion, HE: Hip extension, HF: Hip Flexion. Absolute peak moment parameters were reported as "Nm". Peak moment parameters were also normalized with body weight and reported as "Nm/kg". Asterisk * indicated $\mathrm{p}<0.05$.

The results of the Mann Whitney U analysis revealed that mean declines in work, power and moment generated during 50 repetitions of isokinetic fatigue test were found significantly different between healthy and injured ballet dancers. Work, power and moment parameters in injured ballet dancers were found significantly lower between the repetitions of 1-to 5, 21-to-25 and last five repetitions of isokinetic fatigue test compared to health control group (Table 3 ).

Table 3. Work, power and mean moment parameters generated during 50 repetitions of an endurance test $(n=21)$.

\begin{tabular}{|c|c|c|c|c|c|c|c|}
\hline \multirow[t]{2}{*}{$\begin{array}{l}\text { Endurance } \\
\text { Measures } \\
\end{array}$} & & \multicolumn{2}{|c|}{$\begin{array}{c}\text { Repetitions } \\
1-5 \\
\end{array}$} & \multicolumn{2}{|c|}{$\begin{array}{c}\text { Repetitions } \\
21-25 \\
\end{array}$} & \multicolumn{2}{|c|}{$\begin{array}{c}\text { Last } 5 \\
\text { Repetitions }\end{array}$} \\
\hline & & Extension & Flexion & Extension & Flexion & Extension & Flexion \\
\hline \multirow{2}{*}{ Work (Joules) } & Control & $70.16 \pm 13.73$ & $34.92 \pm 11.23$ & $50.82 \pm 10.56$ & $19.60 \pm 5.15$ & $35.96 \pm 6.85$ & $10.46 \pm 4.66$ \\
\hline & Injured & $55.15 \pm 10.43 * *$ & $25.80 \pm 10.12 *$ & $41.70 \pm 9.47 *$ & $12.50 \pm 5.15^{*}$ & $30.62 \pm 6.85^{*}$ & $8.57 \pm 2.25$ \\
\hline \multirow{2}{*}{ Power (Watt) } & Control & $228.94 \pm 41.47$ & $62.28 \pm 16.15$ & $166.46 \pm 33.37$ & $36.22 \pm 8.81$ & $112.88 \pm 18.74$ & $21.58 \pm 9.07$ \\
\hline & Injured & $198.42 \pm 35.87 * *$ & $51.17 \pm 11.12 * *$ & $129.38 \pm 28.21 * *$ & $30.17 \pm 7.56^{*}$ & $100.13 \pm 11.41 *$ & $16.53 \pm 11.23^{*}$ \\
\hline \multirow{2}{*}{ Moment (Nm) } & Control & $73.86 \pm 13.22$ & $38.04 \pm 9.14$ & $53.80 \pm 10.72$ & $24.92 \pm 6.64$ & $36.62 \pm 6.08$ & $16.32 \pm 5.20$ \\
\hline & Injured & $61.27 \pm 11.26^{* *}$ & $30.42 \pm 10.25^{*}$ & $48.21 \pm 9.56^{*}$ & $19.52 \pm 7.58^{*}$ & $29.21 \pm 8.35^{*}$ & $10.51 \pm 6.27^{*}$ \\
\hline
\end{tabular}

To determine the magnitude of injury on fatigue test performance mean declines in work, power and moment occurred at specified intervals during 50 repetitions of isokinetic fatigue test was found as follows. Percent decline in work, power and moment parameters in injured ballet dancers were found significantly lower between the repetitions from 1-5 to 21-to-25 and 1-5 to last five repetitions of isokinetic fatigue test compared to health control group (Table 4). 
Table 4. Percent decline in mean work, power and moment parameters during 50 repetitions of an endurance test

\begin{tabular}{|c|c|c|c|c|c|}
\hline \multirow[t]{2}{*}{$\begin{array}{l}\text { Percent Decline } \\
(\%)\end{array}$} & & \multicolumn{2}{|c|}{$\begin{array}{l}\text { Repetitions 1-5 to } \\
\text { Repetitions 21-25 }\end{array}$} & \multicolumn{2}{|c|}{$\begin{array}{c}\text { Repetitions } 1-5 \text { to Last } \\
5 \text { Repetitions } \\
\end{array}$} \\
\hline & & Extension & Flexion & Extension & Flexion \\
\hline \multirow{2}{*}{ Work (Joules) } & Control & $15.21 \pm 13.52 * *$ & $35.33 \pm 11.25^{*}$ & $40.28 \pm 6.93^{*}$ & $55.53 \pm 12.21 * *$ \\
\hline & Injured & $27.04 \pm 10.09$ & $42.37 \pm 10.88$ & $48.38 \pm 5.88$ & $67.48 \pm 13.82$ \\
\hline \multirow{2}{*}{ Power (Watt) } & Control & $20.90 \pm 10.21 *$ & $35.03 \pm 14.26^{*}$ & $41.25 \pm 7.37 * *$ & $55.25 \pm 11.43 *$ \\
\hline & Injured & $26.94 \pm 9.42$ & $41.02 \pm 10.40$ & $50.21 \pm 6.25$ & $63.88 \pm 14.08$ \\
\hline \multirow{2}{*}{ Moment (Nm) } & Control & $20.21 \pm 10.01 *$ & $29.35 \pm 11.12^{*}$ & $40.21 \pm 7.35^{*}$ & $50.52 \pm 10.07 *$ \\
\hline & Injured & $26.77 \pm 9.85$ & $34.15 \pm 12.70$ & $49.92 \pm 6.59$ & $56.60 \pm 11.90$ \\
\hline
\end{tabular}

The decline in anaerobic power capacity during Wingate test was reported as shown below. The specified declines at each intervals showed

Table 5. The changes in power parameters during WAnT test (Mean $\pm \mathrm{SD})$.

\begin{tabular}{lcccccc}
\hline & & $\begin{array}{c}\text { Peak power } \\
\text { (Watt) }\end{array}$ & $\begin{array}{c}\text { Average power } \\
\text { (Watt) }\end{array}$ & $\begin{array}{c}\text { Minimum } \\
\text { power (Watt) }\end{array}$ & $\begin{array}{c}\text { Percent } \\
\text { Decline (\%) }\end{array}$ & $\begin{array}{c}\text { Power drop } \\
\text { (Watt) }\end{array}$ \\
\hline \multirow{2}{*}{ Power (Watt) } & Control & $293.00 \pm 75.47$ & $251.48 \pm 51.50$ & $97.51 \pm 45.53$ & $66.72 \pm 32.10$ & $195.49 \pm 83.23$ \\
& Injured & $279.93 \pm 70.34 * *$ & $204.53 \pm 54.59 * *$ & $81.63 \pm 73.48 * *$ & $72.42 \pm 32.10 *$ & $198.29 \pm 83.23$ \\
\hline
\end{tabular}

Note. $* \mathrm{p}<0.05, * * \mathrm{p}<0.001$

\section{Correlations among lower extremity strength characteristics:}

There was negative significant correlations among body weight, $60 \%$ relative left dorsiflexion and $180^{\circ}$ relative right plantar flexion $(\mathrm{r}=.736, \mathrm{p}<.001 ; \mathrm{r}=.713, \mathrm{p}<.001)$ and also H:Q Ratio and non-dominant limb $60 \%$ relative knee flexion, right leg $60 \%$ relative knee extension, and left leg $60 \%$ relative knee flexion strength $(\mathrm{r}=.60, \mathrm{p}<.05 ; \mathrm{r}=.825, \mathrm{p}<.001 ; \mathrm{r}=.701, \mathrm{p}<.05)$, respectively. Additionally, there was also a negative correlation between mean anaerobic significant changes between injured and healthy control group (Table 5).

Table 6. The correlation between isokinetic peak moment, body weight and H:Q ratio parameters.

\begin{tabular}{|c|c|c|c|c|c|}
\hline Variable & $\begin{array}{c}\text { Body Weight } \\
(\mathrm{kg})\end{array}$ & $\begin{array}{c}\mathbf{H}: \mathbf{Q} \\
(\%)\end{array}$ & $\begin{array}{l}\text { WAnT Mean } \\
\text { Power } \\
\text { (Watt) }\end{array}$ & $\begin{array}{c}\text { Extension } \\
\text { Fatigue Index } \\
(\%)\end{array}$ & $\begin{array}{c}\text { Flexion Fatigue } \\
\text { Index } \\
(\%)\end{array}$ \\
\hline$\overline{\mathbf{D F}}^{\text {Left }} \mathbf{6 0} / \mathrm{sec}(\mathrm{Nm} / \mathrm{kg})$ & $-0.736 * *$ & & & & \\
\hline PF $^{\text {Right }} \mathbf{1 8 0} / \mathrm{sec}(\mathrm{Nm} / \mathrm{kg})$ & $-0.713^{* *}$ & & $-.635^{*}$ & & \\
\hline $\mathbf{K F}^{\text {Left }} \mathbf{6 0} / \mathbf{s e c}(\mathrm{Nm} / \mathrm{kg})$ & & $-0.602 *$ & & & \\
\hline $\mathbf{K E}^{\text {Right }} \mathbf{6 0} / \mathrm{sec}(\mathrm{Nm} / \mathrm{kg})$ & & $-0.825 * *$ & & & \\
\hline $\mathbf{K E}^{\text {Left }} \mathbf{6 0} / \mathbf{s e c}(\mathrm{Nm} / \mathrm{kg})$ & & $-0.701 *$ & & & \\
\hline $\mathrm{KE}^{\mathrm{MWD}} 1-5$ to 46-50 (reps) & & & & $.831 * *$ & \\
\hline $\mathrm{KE}^{\mathrm{MPD}} 1-5$ to $46-50$ (reps) & & & & $.961 * *$ & \\
\hline $\mathrm{KE}^{\mathrm{MMD}} 1-5$ to $46-50$ (reps) & & & & .969 & \\
\hline $\mathrm{KF}^{\mathrm{MWD}} 1-5$ to 46-50 (reps) & & & & & $.818 * *$ \\
\hline $\mathrm{KF}^{\mathrm{MPD}} 1-5$ to $46-50$ (reps) & & & & & $.837 * *$ \\
\hline $\mathrm{KF}^{\mathrm{MMD}} \mathbf{1 - 5}$ to $46-50$ (reps) & & & & & $.931 * *$ \\
\hline
\end{tabular}




\section{DISCUSSION}

Training load is a major component of the process of adaptation of the body functions and it helps athletes to prolong the amount of work of a certain type and intensity during a physical exercise, training session, or training cycle (Terjung, 1995). As a result of this long term adaptive process the organism may show different adaptations to given stimulus and may lead to various injuries. Especially, branches such as aerobic dance requires a precision upon muscle groups of lower extremity to avoid dance-related injuries. Based on the results of the current study it could be speculated that significant discrepancies occurred between dominant and non-dominant dorsiflexor peak muscle strength moments may have resulted from the overloads placed upon a single joint or ballet-specific single-leg dominant movements that comprises the majority of standard daily ballet classes (Table 2). Due to the extent to which ballet-specific single-leg dominant movements during aerobic dance only classes, dancers are mostly prone to limb dominancy and unless they are left-footed, ballet dancers are generally tend to use their left leg as dominant leg during landing following sudden jumps or movements such as pirouette and fouette, and these specific movement combinations constitute a great amount of ballet performance and daily ballet classes. According to the results of the current research, a $48.38 \%$ decline in work, $50.21 \%$ in power, and $49.92 \%$ in mean moment performance during knee extension and a $67.68 \%$ decline in work, $63.88 \%$ in power and $56.60 \%$ in the mean moment occurred in the knee flexion during repetitive reciprocal contractions (Table 4). It may have been a significant indication as to why the ballet dancers suffered from ACL injury during the long term ballet classes. Due to the lack of additional strength programs in ballet curriculum the occurrence of the injury might have been attributed by fatigue and agonist-to-antagonist strength discrepancy within the same limb.

Based on the results of a study, which compares the ground reaction forces during landing en pointe and landing on the full foot, it was reported that following landing en pointe after sudden jumps dancers generated a mean maximum force of $531.14 \mathrm{~N}$ while $735.93 \mathrm{~N}$ subsequent to landing on the full foot and also generated a $72.17 \%$ of the force during landing en pointe compared to landing on the full foot (Chockley, 2008). Consequently, during these specific movements the ankle joint has a wider range of motion in jumps landing on the full foot and especially during en pointe, due to the extreme plantar flexion in the ankle, the surrounding muscles requires extra precision to prevent inflammation of several tendons, especially the peroneals (Ritter and Moore, 2008). Similarly, it was found in another study that both male and female dancers have similar incidence rates of the ankle sprain, and the right leg is predominantly used during single-legged movements to ensure postural sway. As a consequence, the incidences generally occur on the right ankle for both genders (Leanderson et al., 1996). Thus, during the preparation phase of the annual ballet training curriculum, it is essential to optimize the balance between symmetric and asymmetric movement patterns to minimize injury occurrence. It is noteworthy that rather than injury prevention, teachers also need to design training programs that comprise additional strength exercises coupled with ballet classes especially concentrating upon lower extremity muscles since these group of muscles expose overload during the performance. It has been pointed out that a compressive force of up to 12 times the bodyweight is applied to the foot and ankle joints during en pointe movement (Meck et al., 2004). Thus, the limited range of motion during the single-legged jumps and gravitational force could increase the force to the foot and ankle joints compared to the doublelegged jumps and increase the risk of injury. On the other hand, according to the significant negative correlations found between ankle plantar flexion strength and Wingate mean anaerobic power performance it could be speculated that injured dancers were unable to deliver the power to the ergometer pedals produced by the ankle, knee, and hip joints (Table 5). With this in mind, it should be noted that lack of symmetrical strength improvement of lower extremity muscles before return to dance would also cause the lower extremity to expose to excessive forces and motions placed upon the injured joint which could possibly increase the re-injury risk during early dance reintegration.

The results of correlation analysis also showed significant inverse interactions between body weight and relative $60 \% \mathrm{~s}$ left dorsiflexion and $180 \%$ s left plantar-flexion, respectively (Table 
6). It could be speculated from these results that the ankle joints of participants are unable to tolerate the body weights especially during pirouette movement, which performed on the left leg and increase the potential risk of ankle sprain or knee injuries resulted from the improper alignment of ankle joints during this repetitive and exhaustive movement. The studies to date clearly pointed out that due to the overuse of the ankle and knee joints in ballet, the most of the injuries occur in the lower extremity (Garrick and Requa, 1993). Especially, in the development of early-onset arthrosis, joint laxity is one of the most prevalent disorders in ballet dancers (Nechaev and Vasil'ev, 2018). Notably, during some specific movements, the rearfoot undergoes excessive supination which results in increased pressure on the lateral region of the ankle correlated with the "moment-angle" relationship (Fearon et al., 2015; Xia and Rymer, 2004). Thus, it can be asserted that these constant movements which comprise a great deal of ballet performance gradually increase joint loadings in both knee and ankle as the weight increased. According to the results of another study, the girls reach the highest degree of general joint laxity at the ages of 15 (Jansson, 2004). Therefore, especially due to the plantar and dorsiflexion strength deficiency, the ankle surrounding muscles should be strengthen in a symmetrically in order to prevent the injuries when the frequency of the general joint laxity is taken into account at these ages. Besides, it was also reported that during the higher limb velocities ankle weakness specifically appears in dorsiflexor muscles and the strength deficiency appeared in dorsiflexion has an important role underlying poor balance and which may in turn increase the risk of injury during aerobic dance performance (Ikezoe et al., 2003).

\section{CONCLUSION}

Based on the results of the changes in work, power, and moment changes attributed by fatigue in injured dancers during muscle endurance testing, it should be noted that adolescent ballet dancers need to employ additional strength programs to improve dance performance and to prevent the incidences of re-injury resulted from reduced muscular strength and fatigue without interfering with aesthetic demands. An awareness of the necessity of the supplemental strength programs will enable dancers and their teachers to develop new training systems, to employ more effective injury prevention programs, and to improve better physical conditioning strategies. Also, it would be possible to control the development of physical conditioning levels of the dancers with the proper selection of training loads.

\section{REFERENCES}

Chi-Hung, ST., Chan, KM., Chin, MK., and Li, CT. (1994). Isokinetic profile of dorsiflexors and plantar flexors of the ankle - a comparative study of elite versus untrained subjects. $B r \quad J$ Sports Med. 28:1. doi: 10.1136/bjsm.28.1.25

Chockley, C. (2008). Ground Reaction Force Comparison Between Jumps Landing on the Full Foot and Jumps Landing en Pointe in Ballet Dancers. J Dance Med Sci. 12:5-8.

Fearon, C., Doherty, L., and Lynch, T. (2015). How Do I Examine Rigidity and Spasticity? Mov Disord Clin Pract. 2:204. doi: 10.1002/mdc3.12147

Garrick, JG., and Requa, RK. (1993). Ballet injuries. An analysis of epidemiology and financial outcome. Am J Sports Med. 21:58690. doi: 10.1177/036354659302100417

Grossman, G., Krasnow, D., and Welsh, TM. (2005). Effective use of turnout: Biomechanical, neuromuscular, and behavioral considerations. J Dance Educ. 5:15-27.doi:

10.1080/15290824.2005.10387279.

Guidetti, L., Gallotta, MC., Emerenziani, GP., and Baldari, C. (2007). Exercise Intensities during a Ballet Lesson in Female Adolescents with Different Technical Ability. Int J Sports Med. 28:736-42. doi: 10.1055/s-2007-964909

Ikezoe, T., Asakawa, Y., and Tsutou, A. (2003). The relationship between quadriceps strength and balance to fall of elderly admitted to a nursing home. J Phys Ther Sci. 15:75-79. doi: $10.1589 /$ jpts. 15.75

Jansson, A., Saartok, T., Werner, S., and Renstrom, P. (2004). General joint laxity in 1845 Swedish school children of different ages: age- and gender-specific distributions. Acta Paediatric 93:1202-6. doi: 10.1111/j.1651-2227.2004.tb02749.x

Kawabata, Y., Senda, M., Oka, T., Yagata, Y., Takahara, Y., Nagashima, H., and Inoue, H. (2000). Measurement of fatigue in knee 
flexor and extensor muscles. Acta Med Okayama. 54:85-90.

Koutedakis, Y., Hukam, H., Metsios, G., Nevill, A., Giakas, G., and Jamurtas, A. (2007). The effects of three months of aerobic and strength training on selected performance and fitness-related parameters in modern dance students. $J$ Strength Cond Res. 21:808-812. doi: 10.1519/00124278200708000-00026

Leanderson, J., Eriksson, E., Nilsson, C., and Wykman, A. (1996). Proprioception in classical ballet dancers. A prospective study of the influence of an ankle sprain on proprioception in the ankle joint. $A m J$ Sports Med. 24:370-4. doi: 10.1177/036354659602400320

Meck, C., Hess, RA., Helldobler, R., and Roh, J. (2004). Pre-pointe evaluation components used by dance schools. J Dance Med Sci. 8:3-42.

Mertz, L., and Docherty, C. (2012). Self-Described Differences Between Legs in Ballet Dancers: Do They Relate to Postural Stability and Ground Reaction Force Measures? J Dance Med Sci. 16:154-160.

Nechaev, VA., and Vasil'ev, AY. (2018). Imaging of Ballet Artists' Hip Joint Pathology (Literature Review). Radiol Res Pract. 3:12.

Ritter, S., and Moore, M. (2008). The relationship between the lateral ankle sprain and ankle tendinitis in ballet dancers. J Dance Med Sci. 12:23-31.
Stracciolini, A., Hanson, E., Kiefer, AW., Myer, GD., and Faigenbaum, AD. (2016). Resistance training for pediatric female dancers. J Dance Med Sci. 20:64-71. doi: 10.12678/1089-313X.20.2.64

Teitz, CC. (2000). Hip and knee injuries in dancers. J Dance Med Sci. 4:23-29.

Terjung, RL. (1995). Muscle adaptations to aerobic training. Sports Sci Exch. 8:1-4.

Twitchett, E., Koutedakis, Y., and Wyon, MA. (2009). Physiological fitness and professional classical ballet performance: A brief review. J Strength Cond Res. 23:27322740. doi: 10.1519/JSC.0b013e3181bc1749

Xia, R., and Rymer, WZ. (2004). The role of shortening reaction in mediating rigidity in Parkinson's disease. Exp Brain Res. 156:524-528. doi: 10.1007/s00221-0041919-9

Yu-Hyung, P., Yu-mi, K., and Byoung-Hee, L. (2013). An ankle proprioceptive control program improves balance, gait ability of chronic stroke patients. J Phys Ther Sci. 1321-1324. doi: 10.1589/jpts.25.1321

How to cite this article: Umutlu, G. and Arınl, Y. (2021). Segmental Musculoskeletal Examinations of Injured Adolescent Ballet Dancers Mimic a Decreased Strength, Anaerobic Power and Endurance Performance. Int J Disabil Sports Health Sci;4(1):45-53. https://doi.org/10.33438/ijdshs.834111 